\title{
Randomized Controlled Trial on the Efficacy and Safety of Virgin Coconut Oil Compared to Mineral Oil in the Treatment of Uremic Xerosis
}

\author{
Jacqueline Michelle G. de las Alas, ${ }^{1}$ Vanessa M. Carpio, ${ }^{1}$ Marissa Elizabeth L. Lim² and Ma. Lorna F. Frez ${ }^{1}$ \\ ${ }^{1}$ Section of Dermatology, Department of Medicine, College of Medicine and Philippine General Hospital, University of the Philippines Manila \\ ${ }^{2}$ Section of Nephrology, Department of Medicine, College of Medicine and Philippine General Hospital, University of the Philippines Manila
}

\begin{abstract}
Background. Uremic xerosis is the most common dermatologic condition present in patients with chronic kidney disease. Emollients were shown to be beneficial and are considered to be the first-line of treatment.

Objective. To assess the efficacy and safety of virgin coconut oil (VCO) compared to mineral oil as a therapeutic moisturizer for uremic xerosis.

Methods. Adult patients undergoing hemodialysis who have uremic xerosis were randomized using a computer-generated list and were instructed to apply either VCO or mineral oil twice daily on the legs for 4 weeks. Primary outcome measures included investigator-assessed clinical efficacy based on overall dry skin score (ODSS), change in skin hydration (corneometer readings), change in skin lipids (sebumeter readings), and quality of life scores. Secondary outcome measures included patientassessed efficacy and advent of adverse effects. Overall therapeutic response was determined in which treatment success was defined as total clearance of xerosis or reduction of ODSS score and increased objective measurements (i.e., corneometer and sebumeter readings) plus moderate to marked patient-assessed efficacy, while treatment failure was defined as failure in any one of these parameters.
\end{abstract}

Results. A total of 45 (22 VCO group, 23 mineral oil group) were recruited and 36 (18 VCO group, 18 mineral oil group) completed the study. The majority of patients in both treatment

\footnotetext{
Paper presented at the Residents Research Forum (Original Paper Category 1st Place), October 2011, Department of Medicine, Philippine General Hospital, University of the Philippines Manila; Residents Research Forum (Experimental Category - 1st Place), November 2011; UP-Philippine General Hospital Expanded Hospital Research Office, Manila; Free paper presentation, February 2012, 20th Regional Conference of Dermatology, Manila; 1st Place - Philippine Society of Nephrology 32nd Annual Convention Research Forum, April 2012, Manila.
}

Corresponding author: Jacqueline Michelle G. de las Alas, MD Section of Dermatology Department of Medicine Philippine General Hospital University of the Philippines Manila

Taft Avenue, Ermita, Manila, 1000 Philippines

Telephone: +632 5548400 local 5105/5106 or 526-2397

Email: jackiedelasalas@gmail.com groups showed improved ODSS, corneometer readings, and quality of life scores. Most patients considered both treatment oils to be moderately to markedly effective. Analysis of overall therapeutic response revealed treatment success of 4 out of 22 in the VCO group and 4 out of 23 in the mineral oil group. VCO demonstrated a trend to benefit in improving xerotic skin (RRR = $1.0 \%, 95 \% \mathrm{Cl}:-30,26.3 ; \mathrm{RR}=0.99,95 \% \mathrm{Cl}: 0.76,1.3)$ but results are inconclusive due to the wide confidence interval.

Conclusion. The application of VCO or mineral oil for 4 weeks may be equally beneficial and safe in improving uremic xerosis. VCO showed a trend to benefit compared to mineral oil in terms of overall therapeutic response but this needs to be confirmed in larger randomized controlled trials.

Key Words: chronic kidney disease, dry skin, uremic xerosis, virgin coconut oil, mineral oil, emollients

\section{Introduction}

Chronic kidney disease (CKD) is among the 10 leading causes of mortality in the Philippines. ${ }^{1}$ According to the Philippine Renal Disease Registry annual report of 2011, a total of 9,133 Filipinos started hemodialysis in 2010 and this incidence was noted to have been steadily increasing in the succeeding years. ${ }^{2}$ In the Philippine General Hospital alone, a total of 25-30 patients undergo hemodialysis per day. ${ }^{3}$ A wide variety of skin diseases can occur in patients with chronic kidney disease. In a study conducted in 2005, Udayakumar and colleagues examined 100 successive patients on hemodialysis and concluded that the top three most prevalent skin conditions included xerosis (79\%), pallor $(60 \%)$, and pruritus $(53 \%){ }^{4}$

Xerosis is predominantly seen over the extensor surfaces of the forearms, legs, and thighs. ${ }^{4}$ It has been attributed to increased levels of vitamin A in the epidermis, atrophy of sebaceous and sweat glands, and dysautonomia. ${ }^{5}$ An association between xerosis and reduced stratum corneum hydration was also demonstrated but the cause of this remains unclear. ${ }^{6}$

The treatment for uremic xerosis has largely been empirical and no treatment has been shown to have sufficient efficacy and safety due to its poorly understood pathophysiological mechanism. However, several studies have demonstrated that emollients may be beneficial in patients with CKD-associated xerosis. $6,7,8$ 
In the Philippines, coconut oil has been used as a traditional moisturizer for centuries. Virgin coconut oil (VCO) is obtained from the fresh, mature kernel of the coconut. It consists mainly of medium-chain triglycerides and fatty acids that can serve as a replacement for lost lipids in the skin's barrier, including lauric, capric, caprylic, and myristic acid. ${ }^{9}$ It also has antiseptic properties. ${ }^{10,11,12}$

A randomized, double-blind controlled trial comparing extra virgin coconut oil with mineral oil as moisturizer for 34 adults with mild to moderate xerosis revealed that both groups resulted in significant improvement in skin hydration and surface lipid levels. The authors concluded that application of VCO was comparable to mineral oil in terms of efficacy and safety when used as a moisturizer. ${ }^{13}$

A randomized controlled community-based trial comparing VCO with mineral oil as therapeutic moisturizer in 148 adults with mild to moderate senile xerosis concluded that VCO showed more beneficial effects than mineral oil in the overall dry skin scores ( $R R R=60.5 \%, 95 \%$ CI: 40.7, 73.7), patient-assessed efficacy $(\mathrm{RRR}=27.6 \%, 95 \% \mathrm{CI}: 15.5,38)$, skin hydration $(\mathrm{RRR}=51.7 \%, 95 \% \mathrm{CI}: 25.8,68.6)$, and skin lipids (RRR $=55.5 \%, 95 \%$ CI: 21.8, 74.6) ${ }^{14}$

An open label clinical trial involving 10 patients who applied VCO twice daily for 1 week for uremic xerosis showed significant post-treatment decrease (Mean pretreatment $=1.8$, Mean post-treatment $=0.8, \mathrm{p}=0.004)$ in uremic xerosis based on xerosis assessment scale. ${ }^{15}$

Given the potential beneficial effects of VCO as an emollient and the need to verify the results of the abovementioned study with a larger controlled randomized clinical trial of longer duration, this study aimed to verify the efficacy and safety of VCO as a therapeutic moisturizer, compared with mineral oil, in adult patients with xerosis associated with a systemic disease, particularly uremia.

\section{Objectives}

\section{General Objective}

To assess the efficacy and safety of virgin coconut oil (VCO) compared to mineral oil as a therapeutic moisturizer for uremic xerosis.

\section{Specific Objectives}

1. To determine and compare changes in the over-all dry skin score (ODSS) within and between treatment groups at baseline, 2-weeks post and 4-weeks post application.

2. To quantitatively measure the following skin parameters at baseline, 2-weeks and 4-weeks post application:

a. Skin hydration using a corneometer

b. Skin lipids using a sebumeter

3. To determine and compare changes in quality of life scores (QoL) within and between treatment groups at baseline and 4-weeks post application.
4. To identify the adverse effects of virgin coconut oil and mineral oil when used as moisturizer for uremic xerosis

\section{Materials and Methods}

\section{A. Study design}

This study was a 4-week, single-center, assessorblinded, randomized controlled trial.

\section{B. Setting and duration of study}

The study was conducted at the University of the Philippines-Philippine General Hospital Section of Nephrology Dialysis Unit. The recruitment began on April 1, 2011 and the last patient follow-up was on July 12, 2011.

\section{Participants}

Adult patients with chronic kidney disease undergoing regular outpatient maintenance hemodialysis who had mild to severe uremic xerosis were recruited and screened for eligibility. Patients with known hypersensitivity to the test oils were excluded as well as patients with open wounds, erosions, and ulcers on the test site, paraplegics and patients with altered sensation on the test sites, and patients with xerosis associated with other dermatologic conditions antedating renal failure.

\section{Sample size calculation}

In order to detect significant difference ( $p$-value $<0.05$ or $5 \%$ level of significance) between the two groups (i.e., if the values of the target variable depends on the group membership of patients) using Repeated Measures Analysis of Variance (ANOVA), it was determined that with an alpha of 0.5 , beta of 0.20 (power $80 \%$ ) the sample size needed per group is at least 25 .

\begin{tabular}{ll}
\hline \multicolumn{1}{c}{ Input } & \multicolumn{1}{c}{ Output } \\
\hline Effect size $\mathrm{f}=0.2$ & Noncentrality parameter \\
& $\lambda=10.0000000$ \\
$\alpha$ err prob $=0.05$ & Critical $\mathrm{F}=3.0911913$ \\
Power $(1-\beta$ err prob $)=0.8$ & Numerator $\mathrm{df}=2.0000000$ \\
Number of groups $=2$ & Denominator $\mathrm{df}=96.0000001$ \\
Number of measurements $=3$ & Total sample size $=50^{*}$ \\
Corr among rep measures $=0.4$ & Actual power $=0.8024486$ \\
\hline
\end{tabular}

${ }^{*}$ which will be divided into 2 groups

\section{E. Study Procedure}

Participants were asked to discontinue application of other topical therapies on the test sites at least 2 weeks prior to study enrollment. Once the informed consent was secured, the primary investigator performed complete history, physical examination and dermatologic evaluation of the eligible participants. Overall dry skin scores (ODSS) ${ }^{16}$ as well as corneometer and sebumeter measurements were 
done. Baseline quality of life scores and photographs of the test areas were also taken.

\section{Randomization and allocation concealment}

After enrollment, patients were then randomized into two treatment groups using a computer-generated list. Treatment allocations (either VCO or mineral oil) were performed by the secondary investigator using numbered, sealed, opaque envelopes.

\section{Test medications}

The VCO (Oleum VCO, Diademoil Corporation, Philippines) was produced through a cold process, without using heat (absolutely no heat or ANH). Cold-processing VCO allows the retention of monoglycerides and other natural antioxidants. It is transparent, colorless, and retains a coconut smell.

The mineral oil (MCB Drug; Manila) is transparent, colorless, odorless oil composed mainly of alkanes and cyclic paraffins.

A third party (research assistant) who was not directly involved in the study placed VCO and mineral oil in identical white plastic bottles which were sequentially numbered and arranged according to the allocation list. The treatment codes were kept in a sealed envelope by the secondary investigator. The code was broken only after the final analysis was completed. The primary investigator who did the assessment was blinded to the treatment allocation. The test oils were visually similar when examined but VCO had a distinct smell, thus making blinding of the patients and caregivers impossible.

Participants were instructed to apply approximately 2 $\mathrm{ml}$ of the assigned test oil to each leg, twice a day (i.e., after bathing in the morning and at bedtime), daily for 4 weeks. An instructional sheet was provided which specified use of any commercially available non-drying soap and avoidance of excessive scrubbing. Application of other emollients was not allowed. Patients were also advised to discontinue test oil application if they felt stinging, burning, erythema, itching, or discomfort.

\section{Assessments}

\section{Dermatology Life Quality Index (DLQI)}

The Dermatology Quality of Life Index (DLQI) questionnaire is a generic skin disease quality of life tool developed by Dr. A.Y. Finlay. ${ }^{17}$ It is made up of 10 questions and can be analyzed under the following headings: symptoms and feelings, daily activities, leisure, work and school, personal relationships, and treatment. It is calculated by summing the score of each question resulting in a maximum of 30 for 10 items and a minimum of 0 . The higher the score, the more quality of life is impaired. The interpretation of the DLQI is as follows:

\begin{tabular}{cl}
\hline Score & \multicolumn{1}{c}{ Interpretation } \\
\hline $0-1$ & No effect at all on the patient's life \\
$2-5$ & Small effect at all on the patient's life \\
$6-10$ & Moderate effect at all on the patient's life \\
$11-20$ & Very large effect at all on the patient's life \\
$21-30$ & Extremely large effect at all on the patient's life \\
\hline
\end{tabular}

The Indeks sa Kalidad ng Pamumuhay ng may Sakit sa Balat or IKPAS is a Filipino translation of Finlay's DLQI questionnaire. ${ }^{18}$ Permission to use the IKPAS was obtained from the author of the Filipino translation. Improved DLQI score was defined as improvement of at least one level in the interpretation of the score (i.e., small effect on patient's life to no effect on patient's life).

\section{F. Outcome measures}

The time point of interest was 28 days after treatment was started.

1. Primary outcomes

a. Clinical efficacy based on the Over-all Dry Skin Score (ODSS)

b. Clinical efficacy rates determined by:

i. Change in skin hydration as measured by corneometer

ii. Change in skin lipids as measured by sebumeter

iii. Change in Quality of Life scores (based on the IKPAS)

\section{Secondary outcomes}

a. Patient assessed clinical efficacy

b. Adverse effects: stinging, burning sensation, redness, pruritus, and discomfort

\section{Determination of Study Outcomes}

The participants were instructed to return for follow-up at the 2nd and 4th weeks. Photographs, ODSS, corneometer and sebumeter readings, and quality of life scores were taken by a blinded assessor at baseline and each follow-up visit. The patient's assessment of efficacy was asked on week 4.

a. Overall Dry Skin Score

\begin{tabular}{ll}
\hline & \multicolumn{1}{c}{ Overall Dry Skin Score } \\
\hline 0 & Absent \\
1 & Fine scaling, faint roughness \& dullness \\
2 & Small scales + few large scales, slight roughness, \\
& whitish appearance \\
3 & Small and large scales uniformly distributed, \\
& $\begin{array}{l}\text { definite roughness, slight redness and few } \\
\text { superficial cracks }\end{array}$ \\
4 & $\begin{array}{l}\text { Dominated by large scales, advanced roughness, } \\
\text { redness, eczematous changes and cracks }\end{array}$ \\
\hline
\end{tabular}


The ODSS was determined by a blinded assessor. The ODSS is a scoring system devised by the EEMCO Group (European Expert Group on Efficacy Measurement of Cosmetics and other Topical products) as practical research tool both for characterization of xerotic skin conditions and for efficacy substantiation of products. ${ }^{16}$ It is a tool widely used internationally in researches involving the clinical assessment of patients with xerosis. Total clearance is defined as ODSS of zero while clinical improvement is defined as at least one point reduction in ODSS from baseline.

b. Objective assessment of efficacy

a. Hydration level of epidermis - as measured by a Corneometer

b. Sebum content of epidermis - as measured by a Sebumeter

c. Corneometer and sebumeter readings were measured using the Multiprobe adapter (MPA; COURAGE-KHAZAKA electronic $\mathrm{GmbH}$, Germany), a plug-in device that combines all skin measurement probes. Readings were obtained on the anterior legs (immediately below the tibial tuberosity). Three measurements were taken for each corneometer and sebumeter reading and were recorded as means and standard deviations. The corneometer measures skin moisture by assessing the in vivo stratum corneum hydration by capacitance measurement. The sebumeter measures the lipid content of the epidermis using the principle of grease-spot photometry. Measurements were taken at weeks 0, 2, and 4. Patient's assessment of efficacy: The investigators asked the patients to assess the efficacy of the study medication at week 4

c. Patient's Assessment of Efficacy

\begin{tabular}{ll}
\hline \multicolumn{2}{c}{ Patient's Assessment of Efficacy } \\
\hline 0 & Not effective \\
1 & Slightly effective \\
2 & Moderately effective \\
3 & Markedly effective \\
\hline
\end{tabular}

Determination of Therapeutic Response

The investigators determined the therapeutic response of participants at weeks 2 and 4 as follows:

1. Treatment success - defined as obtaining all three conditions on the evaluation date:

a. Total clearance or reduction of xerosis based on the ODSS b. Increased skin hydration as measured by corneometer and increased skin lipids as measured by sebumeter

c. Moderate to marked effectiveness based on the patient's assessment of efficacy

2. Treatment failure - defined as satisfying any of the conditions stated below on the evaluation date:

a. No change or increase in ODSS

b. No change or decrease in corneometer or sebumeter measurements

c. No effectiveness or only minimal effectiveness based on patient's assessment of efficacy

\section{G. Data Analysis}

Standard case record forms were used during the data collection. Data was encoded using Microsoft Excel.

\section{Efficacy Analysis}

The primary time point was 4 weeks. Intention to treat (ITT) analysis was the primary efficacy analysis. Per protocol analysis was done as a secondary analysis. Treatment effects were computed using relative risk reduction (RRR), absolute risk reduction (ARR), relative risk (RR), number needed to treat (NNT) with $95 \%$ confidence intervals (CI) based on the primary endpoint, which was treatment failure at 4 weeks. For mean corneometer and sebumeter readings, repeated ANOVA was used to determine significant difference between the two treatment groups.

\section{Safety Analysis}

Adverse events were categorized and presented as frequency distribution. Counting of adverse events was based on the number of subjects and not the number of adverse events.

\section{Handling Missing Data}

Some data was missing due to dropouts or withdrawals. In order to assess the effect of these dropouts and withdrawals at the conclusion of this study, sensitivity analysis using worst-case scenario was performed.

\section{H. Ethical considerations}

This study was conducted in accordance with the guidelines of the Helsinki Declaration, ICH-GCP and was approved by the Philippine General Hospital Expanded Hospital Research Office (EHRO) Ethical Review Board (ERB). Patients were informed of the significance of the study and the risk and benefits of both topical medications was explained. Eligible patients signed a consent form once they have decided to join the study. They were informed that they could withdraw from the study anytime. The identities of the individual patients were not revealed and codes were used to mask their identities. Data gathered was 
reported as group data. Data was kept confidential and was not used for purposes other than this study.

\section{Results}

A total of 61 patients were screened for possible inclusion (Figure 1). Forty-five (45) were randomized into the two treatment groups. There were 3 dropouts in the VCO group and 3 dropouts in the mineral oil group. Forty five (45) patients were included in the intention to treat (ITT) analysis while 39 subjects were included in the per-protocol analysis. Good compliance, based on patient's verbal statement that he or she applied the test oils as instructed, was reported by enrolled patients during the follow-ups. The computed sample size a priori was 25 per group and given the achieved total sample size of 45 ; the post hoc computed achieved power of the test is 0.755 . With a fixed power set at 0.8 , the probability of Type I error is 0.0696 .

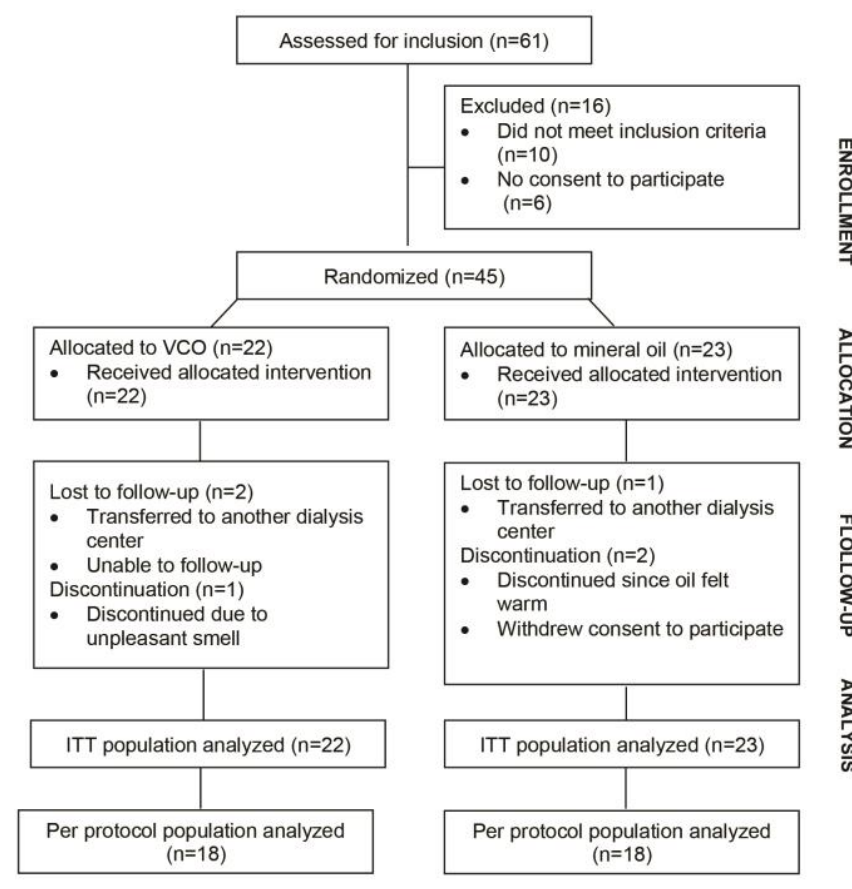

Figure 1. Patient flow chart

There were 22 males (48.9\%) and 23 females (51.5\%) included in the study. The mean age of the subjects was 46 years. There is no significant difference between the baseline characteristics of the two treatment groups (Table 1).

\section{Overall dry skin score (ODSS)}

The distribution of the patients according to ODSS before and after treatment with VCO and mineral oil are seen in Figures 2 and 3, respectively. At baseline, the majority of patients had ODSS of 1 or 2 indicating mild xerosis. A trend towards improvement was noted in both groups from baseline to week 2 and from week 2 to week 4 (Figures 2-5).
Table 1. Baseline characteristics of subjects by treatment group

\begin{tabular}{|c|c|c|}
\hline Parameters & $\begin{array}{c}\text { VCO } \\
\text { Group } \\
(n=22)\end{array}$ & $\begin{array}{c}\text { Mineral Oil } \\
\text { Group } \\
(n=23)\end{array}$ \\
\hline $\begin{array}{l}\text { Total number of subjects who completed the } \\
\text { study (Per protocol population) }\end{array}$ & $18(50 \%)$ & $18(50 \%)$ \\
\hline Mean (SD) age (years) & $46.86(21.03)$ & $45.78(16.16)$ \\
\hline Gender distribution $\mathrm{M} / \mathrm{F}(\%)$ & $\begin{array}{l}10(45.5 \%) / \\
12(54.5 \%)\end{array}$ & $\begin{array}{l}12(52.2 \%) / \\
11(47.8 \%)\end{array}$ \\
\hline Mean (SD) duration of kidney disease (years) & $3.63(3.12)$ & $4.56(3.48)$ \\
\hline $\begin{array}{l}\text { Duration on hemodialysis (years) (median } \\
\text { and interquartile range) }\end{array}$ & $2.29(3.084)$ & $4(5)$ \\
\hline $\begin{array}{l}\text { Baseline ODSS (median and interquartile } \\
\text { range) }\end{array}$ & $2(1)$ & $2(1)$ \\
\hline $\begin{array}{l}\text { Mean (SD) corneometer reading } \\
\text { (Corneometer units) }\end{array}$ & $26.2(9.8)$ & $25.1(8.2)$ \\
\hline Mean sebumeter reading $\left(\mu \mathrm{g} / \mathrm{cm}^{2}\right)$ & $2.8(5.1)$ & $3.2(7.8)$ \\
\hline $\begin{array}{l}\text { Quality of life score (median and } \\
\text { interquartile range) }\end{array}$ & $4(8)$ & $5(5)$ \\
\hline
\end{tabular}

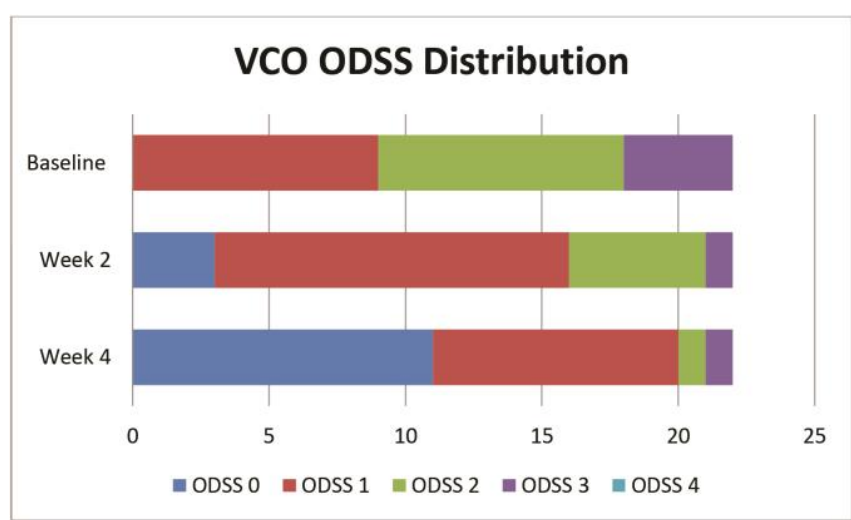

Figure 2. Distribution of the patients according to overall dry skin scores (ODSS), VCO group

\section{Mineral Oil ODSS Distribution}

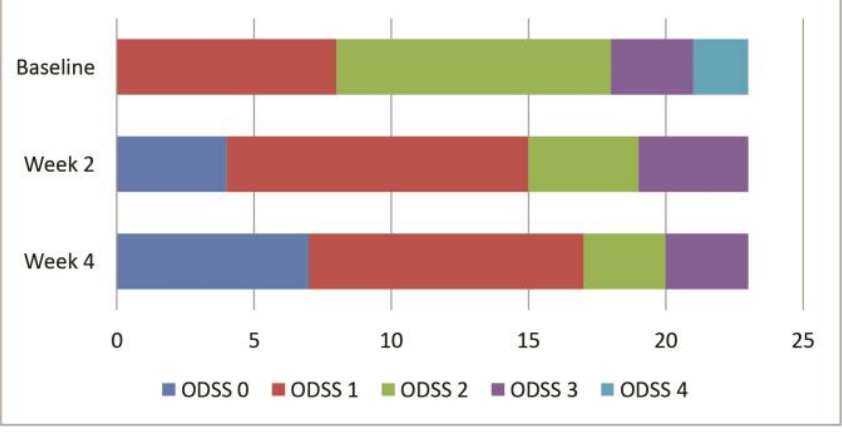

Figure 3. Distribution of the patients according to overall dry skin scores (ODSS), Mineral oil group

After 4 weeks of treatment, $77.3 \%$ of patients in the VCO group achieved clinical improvement of leg xerosis based on ODSS compared to $73.9 \%$ of patients in the mineral oil group (Table 2). Both ITT analysis (RRR = 12.9\%, 95\% CI: -144 , 69; 
$\mathrm{RR}=0.87,95 \% \mathrm{CI}: 0.31,2.45)$ and per protocol analyses (RRR $=50 \%, 95 \% \mathrm{CI}:-403,95 ; \mathrm{RR}=0.5,95 \% \mathrm{CI}: 0.05,5.04)$ of treatment effects for ODSS revealed that VCO showed a trend to benefit compared to mineral oil but this is inconclusive (Tables 3 and 5).

Table 2. Summary of events for VCO versus mineral oil at week 4, per outcome (Intention to treat analysis)

\begin{tabular}{lcccc}
\hline & \multicolumn{2}{c}{ VCO } & \multicolumn{2}{c}{ Mineral oil } \\
\cline { 2 - 5 } & $\begin{array}{c}\text { Failures } \\
\text { No (\%) }\end{array}$ & $\begin{array}{c}\text { Successes } \\
\text { No (\%) }\end{array}$ & $\begin{array}{c}\text { Failures } \\
\text { No (\%) }\end{array}$ & $\begin{array}{c}\text { Successes } \\
\text { No (\%) }\end{array}$ \\
\hline $\begin{array}{l}\text { Overall dry skin score } \\
\text { Patient assessed efficacy }\end{array}$ & $5(22.7)$ & $17(77.3)$ & $6(26.1)$ & $17(73.9)$ \\
$\begin{array}{l}\text { Skin hydration } \\
\text { (Corneometer) }\end{array}$ & $10(45.5)$ & $16(72.7)$ & $7(30.4)$ & $16(69.6)$ \\
$\begin{array}{l}\text { Lipid content } \\
\begin{array}{l}\text { Sebumeter) } \\
\text { Quality of life }\end{array}\end{array}$ & $16(72.7)$ & $6(27.3)$ & $13(56.5)$ & $10(43.5)$ \\
$\begin{array}{l}\text { Overall therapeutic } \\
\text { response }\end{array}$ & $12(54.5)$ & $10(45.5)$ & $9(39.1)$ & $10(43.5)$ \\
\hline
\end{tabular}

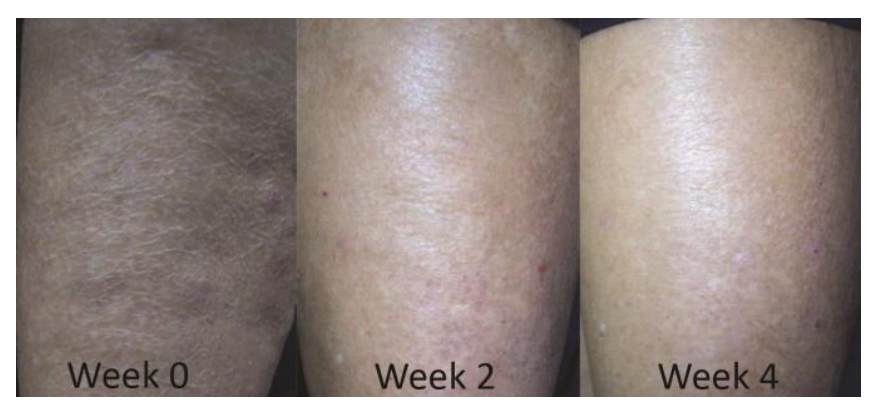

Figure 4. Photographs of lower legs at baseline, week 2, and week 4. (Patient from VCO group)

\section{Patient-assessed efficacy}

At the end of week 4, a total of 16 patients in the VCO group and 16 patients in the mineral oil group perceived the oil to be moderately to markedly effective. Based on ITT analysis seen in Table 3, VCO showed a trend to benefit $(\mathrm{RRR}=10.4 \%, 95 \% \mathrm{CI}:-125,64.3 ; \mathrm{RR}=0.90,95 \% \mathrm{CI}: 0.36$, 2.25) while per protocol analysis revealed a risk difference of zero since both groups had identical results (Tables 4 and 5).

Corneometer readings for skin hydration

More patients in the VCO group had increased corneometer readings from baseline at the end of 4 weeks
(Table 2). Based on both ITT (RRR $=19.6 \%, 95 \%$ CI: $-43.8,55$; $\mathrm{RR}=0.80,95 \% \mathrm{CI}: 0.45,1.44)$ and per-protocol analyses (RRR $=30 \%, 95 \%$ CI: $-42.6,65.6$; RR $=0.7,95 \%$ CI: 0.34, 1.43), VCO showed a trend toward benefit compared to mineral oil but results were inconclusive due to the wide confidence interval (Tables 3 and 5).

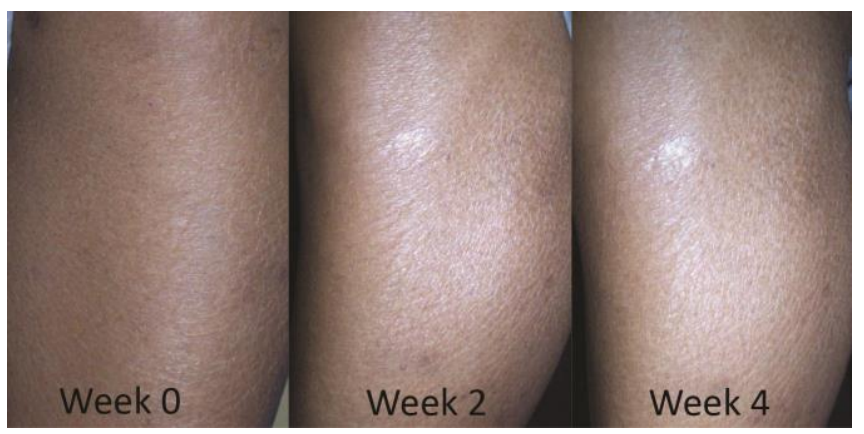

Figure 5. Photographs of lower legs at baseline, week 2, and week 4. (Patient from mineral oil group)

Table 4. Summary of events per outcome, VCO versus mineral oil at week 4 (per-protocol analysis)

\begin{tabular}{lcccc}
\hline & \multicolumn{2}{c}{ VCO } & \multicolumn{2}{c}{ Mineral oil } \\
\cline { 2 - 5 } & $\begin{array}{c}\text { Failures } \\
\text { No (\%) }\end{array}$ & $\begin{array}{c}\text { Successes } \\
\text { No }(\%)\end{array}$ & $\begin{array}{c}\text { Failures } \\
\text { No }(\%)\end{array}$ & $\begin{array}{c}\text { Successes } \\
\text { No (\%) }\end{array}$ \\
\hline $\begin{array}{l}\text { Overall dry skin score } \\
\text { Patient assessed efficacy }\end{array}$ & $1(5.6)$ & $17(94.4)$ & $2(11.1)$ & $16(88.9)$ \\
$\begin{array}{l}\text { Skin hydration } \\
\text { (Corneometer) }\end{array}$ & $7(38.9)$ & $16(88.9)$ & $2(11.1)$ & $16(88.9)$ \\
$\begin{array}{l}\text { Lipid content (Sebumeter) } \\
\text { Quality of life }\end{array}$ & $12(66.7)$ & $6(33.3)$ & $10(55.6)$ & $8(44.4$ \\
$\begin{array}{l}\text { Overall therapeutic } \\
\text { response }\end{array}$ & $9(50)$ & $9(50)$ & $10(55.6)$ & $8(44.4)$ \\
\hline
\end{tabular}

\section{Sebumeter readings for lipid content}

At the end of the trial, $27.3 \%$ of patients in the VCO group had increased sebumeter readings from baseline, compared to $43.5 \%$ of patients in the mineral oil group (Table 2). Both ITT (RRR = -28.7\%, 95\% CI: $-999,17.2 ; \mathrm{RR}=$ 1.29, 95\% CI: $0.83,1.99)$ and per-protocol analyses (RRR $=-$ 20\%, 95\% CI: $-103,29.1$; RR $=1.2,95 \%$ CI: 0.71, 2.03) revealed that VCO showed a trend toward harm compared to mineral oil (Tables 3 and 5); however, this is inconclusive.

Table 3. Summary of treatment effects per outcome: VCO versus mineral oil at week 4 (Intention to treat analysis)

\begin{tabular}{|c|c|c|c|c|c|c|c|c|}
\hline & RRR (\%) & $95 \% \mathrm{CI}$ & ARR (\%) & $95 \% \mathrm{CI}$ & RR & $95 \% \mathrm{CI}$ & NNT & $95 \% \mathrm{CI}$ \\
\hline Overall dry skin score & 12.9 & $-144,69$ & 3.4 & $-21.4,27.3$ & 0.87 & $0.31,2.45$ & 29 & $-5,4$ \\
\hline Patient Assessment & 10.4 & $-125,64.3$ & 3.2 & $-22.4,28$ & 0.90 & $0.36,2.25$ & 31 & $-4,4$ \\
\hline Skin hydration & 19.6 & $-43.8,55$ & 11.1 & $-16.9,36.8$ & 0.80 & $0.45,1.44$ & 9 & $-6,3$ \\
\hline Lipid content & -28.7 & $-99.9,17.2$ & -16.2 & $-40.5,11.3$ & 1.29 & 0.83 .1 .99 & NNH 7 & $-2,9$ \\
\hline Quality of life & -39.4 & $-163,26.3$ & -15.4 & $-40.5,12.8$ & 1.39 & $0.74,2.64$ & NNH 7 & $-2,8$ \\
\hline Over-all therapeutic response & 1.0 & $-30,24.5$ & 0.8 & $-21.8,23.6$ & 0.99 & $75.5,1.30$ & 126 & $-5,4$ \\
\hline
\end{tabular}


VCO vs. Mineral Oil for Uremic Xerosis

Table 5. Summary of treatment effects per outcome: VCO versus mineral oil at week 4 (per-protocol analysis)

\begin{tabular}{|c|c|c|c|c|c|c|c|c|}
\hline & RRR & $95 \% \mathrm{CI}$ & ARR & $95 \% \mathrm{CI}$ & RR & $95 \% \mathrm{CI}$ & NNT & $95 \% \mathrm{CI}$ \\
\hline Parameter & $\%$ & & $\%$ & & & & & \\
\hline Overall dry skin score & 50 & $-403,95$ & 5.6 & $-16.2,27.7$ & 0.5 & $0.05,5.04$ & 18 & $-6,4$ \\
\hline Patient Assessment & - & - & 0 & - & 1 & - & - & - \\
\hline Skin hydration & 30 & $-42.6,65.6$ & 16.7 & $-14.7,49.9$ & 0.7 & $0.34,1.43$ & 5 & $-7,2$ \\
\hline Lipid content & -20 & $-103,29.1$ & -11.1 & $-38.8,19.2$ & 1.2 & $0.71,2.03$ & NNH 10 & $-3,5$ \\
\hline Quality of life & -125 & $-499,15.6$ & -27.8 & $-52.6,3.5$ & 2.25 & $0.84,5.99$ & NNH 4 & $-2,30$ \\
\hline Over-all therapeutic response & - & - & 0 & - & 1 & - & - & - \\
\hline
\end{tabular}

\section{Quality of life measurements}

Baseline scores in both groups demonstrated that uremic xerosis had moderately affected the patient's quality of life with a mean score of 5.14 and 7.17 in the VCO and mineral groups, respectively. All scores at end of treatment were significantly lower from baseline $(p$-value $=0.000)$ within groups.

At the end of four weeks, 10 out of 22 (45.5\%) of patients in the VCO group had improved quality of life (i.e., decrease of at least one level in the interpretation of the score) from baseline, compared to 14 out of $23(60.9 \%)$ patients in the mineral oil group (Table 2). Based on ITT (RRR $=-39.4 \%$, 95\% CI: $-163,26.3$; RR $=1.39,95 \% \mathrm{CI}: 0.74,2.64)$ and perprotocol analyses $(\mathrm{RRR}=-125,95 \% \mathrm{CI}:-499,15.6$; $\mathrm{RR}=2.25$, 95\% CI: 0.84, 5.99), VCO showed a trend to harm in improving uremic xerosis based on improvement of quality of life scores but results were inconclusive due to the wide confidence interval (Tables 3 and 5).

\section{Overall therapeutic response}

Treatment success was achieved by 4 out of 22 patients in the VCO group and 4 out of 23 patients in the mineral oil group. Analysis of overall therapeutic response was computed using the clinical endpoint of failure at 4 weeks. Based on ITT, VCO showed a trend toward benefit in improving xerotic skin ( RRR $=1.0 \%, 95 \% \mathrm{CI}:-30,26.3$; $\mathrm{RR}=$ 0.99, 95\% CI: 0.76, 1.3) (Table 3). Per protocol analysis revealed a risk difference of zero since both groups had identical results (Tables 4 and 5).

\section{Sensitivity analysis/Worst case scenario}

Sensitivity analysis was performed to determine the effect of the drop-out rates on efficacy rates. The VCO group had 3 drop-outs (13.6\%) while the mineral oil group had 3 drop-outs $(13 \%)$. In the worst case scenario analysis, it is assumed that drop-outs in the VCO group are treatment failures, while drop-outs in the mineral oil group achieved treatment success. Using the worst case scenario sensitivity analysis of the overall therapeutic response, VCO showed a trend to harm compared to mineral oil (RRR $=-21.4 \%, 95 \%$ CI: $-75.2,15.9$; $R R=1.21,95 \% \mathrm{CI}: 0.84,1.75)$. These findings were in contrast with the intention to treat analysis. The dropouts may have had an impact on the study conclusion.

\section{Adverse events}

Adverse events were comparable in both groups, reported by 3 patients in the VCO group and by 4 patients in the mineral oil group. There were no reported serious adverse events. Noted adverse events were mild and symptomatic. Among the reported events, the unpleasant smell of VCO and the warm feeling after application of mineral oil warranted discontinuation of one patient in each treatment group.

\section{Discussion}

Advances in medical and surgical management of chronic kidney disease have led to prolonged life expectancy among patients with end-stage renal disease. This increase in survival rate has led to the occurrence of other symptoms, such as xerosis associated with kidney disease, that may further decrease the quality of life of these patients.

At baseline, most of the patients in this study had mild uremic xerosis but this still had a moderate impact on their quality of life based on the patients' DLQI questionnaire mean scores of 5.14 and 7.17 in the VCO and mineral groups, respectively. This effect was seen in the symptoms and feelings subscale. These results support with findings in previous reports that uremic xerosis indeed has a significant psychosocial impact on dialysis patients.

This trial demonstrated that in majority of patients, both test oils have shown decreased ODSS, increased corneometer readings for skin hydration, increased quality of life scores, and moderate-to-marked patient-assessed efficacy after 4 weeks of treatment. These findings further establish that the use of emollients may improve both objective and subjective symptoms of uremic xerosis. One mechanism through which emollients work is by providing an occlusive layer that reduces transepidermal water loss. Both mineral oil and VCO may act through this mechanism thus making them efficacious for uremic xerosis.

Of note is the minimal improvement in the skin lipids measured using the sebumeter in both treatment groups. This result may be due to the possibility that the sebumeter measures mostly surface lipids secreted by the sebaceous glands. However in patients undergoing hemodialysis, atrophy of the sebaceous glands leading to decreased surface lipids is believed to be part of the pathogenesis of uremic xerosis. ${ }^{5}$ This pathology is unlikely to be improved with 
emollient therapy. Lipids within the stratum corneum's lipid lamellae, and not surface lipids, are thought to be targeted and most likely replaced by therapy with oil-based emollients hence the minimal improvement in sebumeter readings.

Intention to treat analysis of overall therapeutic response showed revealed that VCO showed a trend to benefit based on the criteria of treatment success. Although this is inconclusive due to the wide confidence interval, in theory the physiological lipids such as free fatty acids in VCO may ease the penetration of ingredients into the stratum corneum and help reform the skin's lipid lamellae, something which non-physiological lipids like mineral oil are unable to do. ${ }^{19}$ Larger randomized controlled trials are recommended to confirm this trend.

\section{Conclusions and Recommendations}

There is a trend towards benefit for VCO compared to mineral oil in terms of overall therapeutic response but is inconclusive, and needs to be confirmed in larger randomized controlled trials. Since both oils improved overall dry skin scores, corneometer readings, and the patient's quality of life, the use of either emollient may therefore be recommended in the management of uremic xerosis.

This study was not able to achieve its target sample size due to the limited number of hemodialysis patients that can be accommodated in the institution's hemodialysis unit. A trial involving multiple centers to enable recruitment of a larger population of patients or a longer recruitment period to further validate the results of this study is suggested. Measurement of transepidermal water loss (TEWL), together with corneometer and sebumeter measurements, is also recommended to determine if the test oils are effective in improving the stratum corneum's barrier function. Trials using other emollients containing physiological lipids or humectants for uremic xerosis are also suggested. Lastly, future studies may be conducted on the use of VCO or mineral oil for other chronic dry skin disorders caused by systemic illness.

\section{Acknowledgments}

This study was partially funded by the Philippine Dermatological Society Research Grant.

\section{References}

1. Republic of the Philippines Department of Health, Leading causes of mortality [Online]. 2009 [cited 2013 Jan]. Available from http://www.doh.gov.ph/node/198.html.

2. Republic of the Philippines Department of Health, New rules and regulations regarding the licensure and regulation of dialysis facilities in the Philippines [Online]. 2012 [cited 2013 Jan]. Available from http://www.bhfs.doh.gov.ph/images/issuances/dialysis/ao2012-0001.pdf.

3. University of the Philippines-Philippine General Hospital Department of Medicine Section of Nephrology Year End Report, 2009.

4. Udayakumar P, Balasubramanian S, Ramalingam KS, Lakshmi C, Srinivas CR, Mathew AC. Cutaneous manifestations in patients with chronic renal failure on hemodialysis. Indian J Dermatol Venereol Leprol. 2006;72(2):119-25.

5. Akhyani M, Ganji MR, Samadi N, Khamesan B, Daneshpazhooh M. Pruritus in hemodialysis patients. BMC Dermatol. 2005; 5:7.

6. Morton CA, Lafferty M, Hau C, Henderson I, Jones M, Lowe JG. Pruritus and skin hydration during dialysis. Nephrol Dial Transplant.1996;11(10):2031-6.

7. Okada K, Matsumoto K. Effect of skin care with an emollient containing a high water content on mild uremic pruritus. Ther Apher Dial. 2004; 8(5):419-22

8. Szepietowski JC, Szepietowski T, Reich A. Efficacy and tolerance of the cream containing structured physiological lipids with endocannabinoids in the treatment of uremic pruritus: A preliminary study. Acta Dermatovenereol Croat. 2005; 13(2):97-103.

9. Philippine National Standards (PNS) for Virgin Coconut Oil Report No: PNS/BAFPS 22: 2004.Manila: Bureau of Product Standards (Philippines); 2004. p. 8.

10. Verallo-Rowell VM. Rx: Coconuts! (the perfect Health Nut):How the Coconut Can Enhance Your Life and Well-Being. Indiana, USA: Xlibris Corporation; 2004.

11. Carpo BG, Verallo-Rowell VM, Kabara JJ. Novel antibacterial activity of monolaurin compared with conventional antibiotics against organisms from skin infections: an in vitro study. J Drugs Dermatol. 2007; 6(10):991-8.

12. Abraham ERL, Verallo-Rowell VM. Safety and efficacy of monolaurin, a coconut oil extract versus ethyl alcohol in rinse-free hand antiseptic gells on MMC personnel's hands and microbial isolates. J Phil Dermatol Soc. 2001; 10:90-9.

13. Agero AL, Verallo-Rowell VM. A randomized double-blind controlled trial comparing extra virgin coconut oil with mineral oil as a moisturizer for mild to moderate xerosis. Dermatitis. 2004; 15(3):109-16.

14. Escuadro MO, Dofitas BL. A randomized double-blind controlled trial on the efficacy and safety of virgin coconut oil versus mineral oil as therapeutic moisturizer for senile xerosis. 2009. Unpublished.

15. Aquino RC, Montemayor ES. Virgin coconut oil for uremic pruritus and xerosis: An open labeled paired comparison study. 2006. Unpublished.

16. Serup J. EEMCO guidance for the assessment of dry skin (xerosis) and ichthyosis: clinical scoring systems. Skin ResTechnol. 1995; 1(3):109-14.

17. Finlay AY, Khan GK. Dermatology Life Quality Index (DLQI) - a simple practical measure for routine clinical use. Clin Exp Dermatol. 1994; 19(3):210-6.

18. Santos M, Balagat R, Frez ML. The impact of skin disease on the Quality of Life of patients at the Philippine General Hospital Outpatient Clinic. J Phil Dermatol Soc. 2005; 18(1):9-13.

19. Proksch E, Lachapelle JM. The management of dry skin with topical emollients - recent perspectives. J Dtsch Dermatol Ges. 2005; 3(10):76874. 